\title{
Multispectral optoacoustic tomography of peripheral arterial disease based on muscle hemoglobin gradients-a pilot clinical study
}

\author{
Angelos Karlas ${ }^{1,2,3,4}$, Max Masthoff ${ }^{\sharp \#}$, Michael Kallmayer ${ }^{3}$, Anne Helfen ${ }^{5}$, Michail Bariotakis ${ }^{1,2}$, \\ Nikolina Alexia Fasoula ${ }^{1,2}$, Michael Schäfers ${ }^{6}$, Max Seidensticker ${ }^{7}$, Hans-Henning Eckstein ${ }^{3,4}$, \\ Vasilis Ntziachristos $^{1,2,4 *}$, Moritz Wildgruber ${ }^{5,7 *}$
}

${ }^{1}$ Chair of Biological Imaging, School of Medicine, Technical University of Munich, Munich, Germany; ${ }^{2}$ Institute of Biological and Medical Imaging, Helmholtz Zentrum München (GmbH), Neuherberg, Germany; ${ }^{3}$ Clinic of Vascular and Endovascular Surgery, Technical University of Munich, Klinikum rechts der Isar, Munich, Germany; ${ }^{4}$ DZHK (German Centre for Cardiovascular Research), partner site Munich Heart Alliance, Munich, Germany; ${ }^{5}$ Department for Clinical Radiology, University Hospital Münster, Münster, Germany; ${ }^{6}$ Department for Nuclear Medicine and European Institute for Molecular Imaging, University Hospital Münster, Münster, Germany; ${ }^{7}$ Department for Radiology, University Hospital, LMU Munich, München, Germany

Contributions: (I) Conception and design: A Karlas, M Masthoff, A Helfen, M Bariotakis, M Wildgruber; (II) Administrative support: M Kallmayer, M Schäfers, HH Eckstein, V Ntziachristos, M Wildgruber; (III) Provision of study materials or patients: A Karlas, M Masthoff, M Kallmayer, A Helfen, NA Fasoula, HH Eckstein, M Wildgruber; (IV) Collection and assembly of data: A Karlas, M Masthoff, A Helfen, M Wildgruber; (V) Data analysis and interpretation: A Karlas, M Masthoff, A Helfen, M Bariotakis, NA Fasoula, V Ntziachristos, M Wildgruber; (VI) Manuscript writing: All authors; (VII) Final approval of manuscript: All authors.

"These authors contributed equally to this work.

*These authors contributed equally to this work.

Correspondence to: Moritz Wildgruber, MD, PhD. Department of Radiology, Klinikum der Ludwig-Maximilians-Universität München, Marchioninistrasse 15, 81377 München, Germany, Email: moritzwildgruber@web.de.

Background: Current imaging assessment of peripheral artery disease (PAD) relies on anatomical crosssectional visualizations of the affected arteries. Multispectral optoacoustic tomography (MSOT) is a novel molecular imaging technique that provides direct and label-free visualizations of soft tissue perfusion and oxygenation.

Methods: MSOT was prospectively assessed in a pilot trial in healthy volunteers (group $\mathrm{n}_{1}=4$, mean age $31,50 \%$ male and group $n_{3}=4$, mean age $37.3,75 \%$ male) and patients with intermittent claudication (group $\mathrm{n}_{2}=4$, mean age $72,75 \%$ male, PAD stage $\mathrm{IIb}$ ). We conducted cuff-induced ischemia (group $\mathrm{n}_{1}$ ) and resting state measurements (groups $\mathrm{n}_{2}$ and $\mathrm{n}_{3}$ ) over the calf region. Spatially resolved mapping of oxygenated $\left(\mathrm{HbO}_{2}\right)$, deoxygenated $(\mathrm{Hb})$ and total $(\mathrm{THb})$ hemoglobin, as well as oxygen saturation $\left(\mathrm{SO}_{2}\right)$, were measured via hand-held hybrid MSOT-Ultrasound based purely on hemoglobin contrast.

Results: Calf measurements in healthy volunteers revealed distinct dynamics for $\mathrm{HbO}_{2}, \mathrm{Hb}, \mathrm{THb}$ and $\mathrm{SO}_{2}$ under cuff-induced ischemia. $\mathrm{HbO}_{2}, \mathrm{THb}$ and $\mathrm{SO}_{2}$ levels were significantly impaired in PAD patients compared to healthy volunteers $(\mathrm{P}<0.05$ for all parameters). Revascularization led to significant improvements in $\mathrm{HbO}_{2}$ of the affected limb.

Conclusions: Clinical MSOT allows for non-invasive, label-free and real-time imaging of muscle oxygenation in health and disease with implications for diagnostics and therapy assessment in PAD.

Keywords: Medical imaging; photoacoustic techniques; peripheral arterial disease

Submitted Apr 14, 2020. Accepted for publication Sep 18, 2020.

doi: $10.21037 /$ atm-20-3321

View this article at: http://dx.doi.org/10.21037/atm-20-3321 


\section{Introduction}

Intermittent claudication, the clinical hallmark of peripheral artery disease (PAD), is the result of impaired tissue perfusion and oxygenation due to partial or complete arterial obstruction in the affected limb (1). PAD is a common disease, associated with serious complications such as impaired walking endurance due to pain, development of chronic wounds with poor wound healing and increased risk for infections, decrease in quality of life or even limb amputation. Furthermore, PAD is known to increase the risk for cardiovascular events and death (2).

Diagnosis and grading of PAD includes clinical assessment and peripheral blood pressure assessments in form of anklebrachial index (ABI) before and after treadmill exercise (3). Besides clinical assessment, PAD evaluation is based on noninvasive imaging playing a key role for the characterization of PAD and subsequent therapy planning (4). Current diagnostic strategies are based on duplex ultrasonography (DUS), magnetic resonance angiography (MRA) or computed tomography angiography (CTA). DUS provides tomographic imaging of arterial wall morphology and blood flow profiles of the diseased artery. MRA or CTA produce three-dimensional luminographies of the complete arterial tree of the lower limb, after the administration of contrast agents.

However, the above-mentioned methods provide only indirect assessments of disease stage by visualizing only macrovascular anatomical and hemodynamic information, such as the location and degree of arterial stenosis, without providing direct and objective information about the perfusion and oxygenation of the affected muscle. Therefore, novel diagnostic approaches are being explored to objectively assess muscle perfusion and oxygenation: contrast-enhanced ultrasound (CEUS) requires the intravenous injection of microbubbles to visualize the perfusion of skeletal muscle (5). Moreover, several magnetic resonance techniques have been developed to measure muscle perfusion (arterial spin labeling, ASL) and oxygenation (blood oxygenation-level dependent, BOLD) without the need for exogenous contrast (6). Near-infrared spectroscopy (NIRS) employs light in the near-infrared range (NIR, 680-980 $\mathrm{nm}$ ) to resolve the distributions of oxy- $\left(\mathrm{HbO}_{2}\right)$ and deoxyhemoglobin $(\mathrm{Hb})$ in tissue or, less often, the distribution of the injected indocyanine green (ICG) $(7,8)$. Nevertheless, NIRS perfusion and oxygenation recordings come with high uncertainty about the clear contributions of muscle or superficial soft tissues (especially subcutaneous fat and skin) in the measured signal. Diffuse optical tomography (DOT) employs near-infrared light to record tomographic images of perfusion and oxygenation for several centimeters through the examined tissue (9). However, both NIRS and DOT provide only low spatial resolution images (e.g., 5-10 mm) due to light scattering in tissue, and thus have not entered routine clinical practice.

Multispectral optoacoustic tomography (MSOT) has been introduced to overcome these limitations by taking advantage of the homonymous effect, wherein the emission and absorption of near infrared light pulses lead to the generation of ultrasound waves within the scanned tissue. This way, MSOT produces high-resolution (50-300 $\mu \mathrm{m}$ depending on the setup) images of blood vessels (10-12), as well as tissue perfusion and oxygenation based purely on hemoglobin gradients in space and time $(13,14)$. MSOT has recently shown its unique ability to characterize vascular malformations (15) and to assess perfusion and oxygenation of soft tissues such as skin in systemic sclerosis (16), brown adipose tissue (17), thyroid gland (18), breast cancer tumors (19) and intestines (20) in patients with Crohn's disease. Moreover, tissue perfusion is also disturbed under vascular occlusion conditions. First applications in healthy volunteers showed the potential of MSOT to quantitatively image skeletal muscle perfusion in iatrogenic occlusions of arteries and veins (13).

Thus, we hypothesized in this study that MSOT could also be used for the non-invasive and label-free assessment of skeletal muscle perfusion and oxygenation in PAD. By means of a clinical hybrid MSOT-ultrasound (US) system we measured the $\mathrm{HbO}_{2}$ and $\mathrm{Hb}$ content within the calf muscles of healthy volunteers under cuff-induced conditions of arterial occlusion, as well as of patients with previously diagnosed PAD before and after revascularization therapy.

\section{Methods}

\section{Cuff-induced arterial occlusion measurements}

To investigate the capability of MSOT to accurately image calf muscle perfusion and oxygenation over time, we conducted a set of controlled cuff-induced arterial occlusion measurements in healthy volunteers. Four $\left(n_{1}=4\right.$, 2 females, 2 males, age $31 \pm 3.2$ years) non-smokers, without any vascular or muscular disease were included (Table 1). All participants were asked to consume no caffeine for at least 6 hours before the measurements to avoid its influence on vascular reactivity. Subjects lied in the prone 
Table 1 Detailed information on the four patients and the two sets of four healthy volunteers that participated in the study

\begin{tabular}{lccl}
\hline Group & Age & \multicolumn{1}{c}{ Sex } & Intervention \\
\hline Cuff-occlusions & $31 \pm 3.2$ & 2 females: 2 males & N/A \\
Patients & $72 \pm 5.9$ & 1 female: 3 males & PTA/stenting of a total occlusion of the left common iliac artery \\
& & PTA of the left posterior and the anterior tibial arteries \\
& & PTA of the in-stent stenosis in the right superficial femoral artery \\
& & PTA/stenting of left common iliac artery \\
Healthy & $37.3 \pm 13.9$ & 1 female: 3 males & N/A \\
\hline
\end{tabular}

Patients before intervention were grouped as "Pre-Op", while the same patients after intervention were grouped as "Post-Op". The four healthy volunteers that participated in the occlusion test are grouped as "Occlusion", and those that were used for comparisons with the patients are grouped as "Healthy". PTA, percutaneous transluminal angioplasty.

position and a cuff of appropriate size was placed just above the knee of their dominant leg. The cuff was inflated to $40 \mathrm{mmHg}$ above the systolic blood pressure of each subject. The MSOT-US probe was placed at the level of the largest calf circumference for each subject and kept stable during scanning in a transversal position for 1 minute before cuff inflation, 3 minutes during the occlusion and 2 minutes after cuff deflation. Each cuff measurement lasted for $15 \mathrm{~min}$.

\section{PAD study population and measurements}

Four patients $\left(\mathrm{n}_{2}=4,1\right.$ female, 3 males, age $\left.72 \pm 5.9\right)$ with PAD (Fontaine Stage IIb; Rutherford Grade I, Category 2-3), previously diagnosed by means of US, MRA or CTA were included (Table 1). Patients were asked to lie in supine position with the knees bent. They were then scanned over the affected limb before and $24 \mathrm{~h}$ after the revascularization with balloon and stent angioplasty of the superficial femoral and popliteal artery. No patient suffered from acute limb ischemia at the time point of the preoperative examination. Additionally, four healthy volunteers $\left(\mathrm{n}_{3}=4,1\right.$ female, 3 males, age $37.3 \pm 13.9$ ) were also examined as controls (Table 1). The hand-held scanning probe was placed for both patients and healthy volunteers at the level of the largest calf circumference in a transversal position. Each scan lasted for approximately 10 seconds. Total duration of MSOT examinations was approximately $15 \mathrm{~min}$, which is comparable to ultrasound.

The study was conducted in accordance with the Declaration of Helsinki (as revised in 2013). All participants gave written and informed consent prior to study enrolment. The study was reviewed by the local ethics committee (Ethikkommission der Westfälischen WilhelmsUniversität Münster, Protocol No. 2017-538-f-S).

\section{MSOT setup and image acquisition}

Technical details of the clinical hand-held MSOT-US system (MSOT Acuity Echo, iThera Medical GmbH, Munich) have been previously described (20,21). The central frequency of the ultrasound transducer was $4 \mathrm{MHz}$. The output light pulse energy $(\sim 15 \mathrm{~mJ}$ illuminating an area of $1 \mathrm{~cm} \times 4 \mathrm{~cm}$ ) adhered to the American National Standards Institute (ANSI) limits of maximum permissible human exposure (22). The central frequency MSOT achieved an imaging penetration depth of $\sim 3-4 \mathrm{~cm}$ with a spatial resolution of $\sim 250 \mu \mathrm{m}$. Co-registered US images (8 Hz frame rate) were generated and recorded for all MSOT frames ( $25 \mathrm{~Hz}$ frame rate). Experiments took place at normal room temperature $\left(\sim 22{ }^{\circ} \mathrm{C}\right)$. Both healthy volunteers and patients rested for at least $5 \mathrm{~min}$ before examination.

MSOT images were acquired at multiple different wavelengths in the NIR including 700, 730, 760, 780, 800 and $850 \mathrm{~nm}$, where the $\mathrm{HbO}_{2}$ and the $\mathrm{Hb}$ demonstrate prominent spectral differences. Optoacoustic data for each pulse (wavelength) were reconstructed into tomographic images by means of a customized model-based algorithm (23). Regions of interest (ROIs) were delineated in the US images over the calf muscle in consensus between two examiners blinded for patient or healthy volunteer data. For each subject one characteristic US image was selected based on image quality and low motion artifacts. ROIs were defined based on the local anatomy and the known texture of muscle tissue in US. Levels of $\mathrm{HbO}_{2}, \mathrm{Hb}$, total hemoglobin $(\mathrm{THb})$ and hemoglobin saturation $\left(\mathrm{SO}_{2}\right)$ 

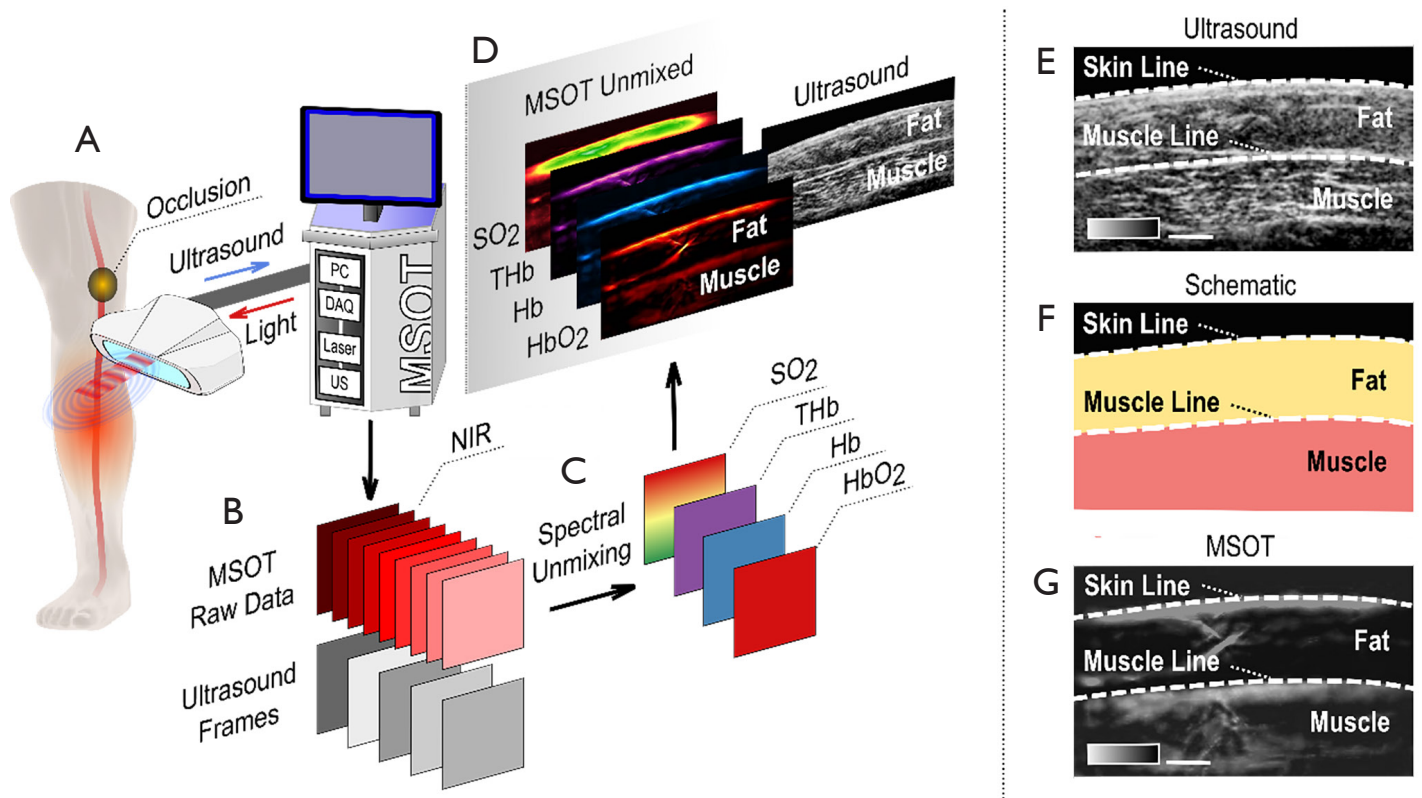

Figure 1 MSOT-US principle of operation and data workflow. (A) Calf muscle is illuminated with pulsed light and US waves are generated upon light absorption. Both illumination and US detection take place by means of the same hand-held MSOT scanner. (B) For each nearinfrared light pulse, an MSOT and a co-registered US frame are recorded. The frame rate is approximately $25 \mathrm{~Hz}$ for MSOT and $8 \mathrm{~Hz}$ for US. (C) The recorded raw MSOT data are further unmixed in $\mathrm{HbO}_{2}$ and $\mathrm{Hb}$ images and the corresponding total hemoglobin THb $\left(\mathrm{HbO}_{2}+\mathrm{Hb}\right)$ and hemoglobin saturation $\mathrm{SO}_{2}\left(\mathrm{HbO}_{2} / \mathrm{THb}\right)$ are calculated. (D) Finally, a set of 5 images $\left(\mathrm{US}, \mathrm{HbO}_{2}, \mathrm{Hb}, \mathrm{THb} \mathrm{SO}\right)$ is produced for the extraction of the image-based biomarkers. (E) Exemplary US image of the calf showing the main anatomical regions: skin surface, subcutaneous fat and muscle area. Upper white dashed line: skin surface. Lower white dashed line: interface between subcutaneous fat and muscle or else muscle line. Muscle area: image area below the muscle line. (F) Schematic of the exemplary US (E) and MSOT (G) images of the calf. (G) Exemplary MSOT image at $800 \mathrm{~nm}$ of the same region as in (E). This representation and annotation are followed throughout the text for all skeletal muscle images. Scale bars: $0.5 \mathrm{~cm}$. NIR, near-infrared range; PC, computer; DAQ, data acquisition card; MSOT, multispectral optoacoustic tomography; US, ultrasonography.

were extracted from corresponding MSOT images via a spectral unmixing algorithm used in previous clinical MSOT studies (17). The THb image was calculated as the sum of the spectrally unmixed $\mathrm{HbO}_{2}$ and $\mathrm{Hb}$ images. The $\mathrm{SO}_{2}$ image was calculated in a per-pixel manner by dividing the $\mathrm{HbO}_{2}$ image by the resulted $\mathrm{THb}$ image. The MSOT principle of operation and the data workflow are graphically presented in Figure 1.

\section{Statistical analysis}

Data were analyzed using the $\mathrm{R}$ environment for statistical analysis and MATLAB ${ }^{\odot}$ (Version R2018b, The MathWorks, Inc., Natick, MA, USA). A two-sided student's $t$-tests was used for group comparisons when data where normal, and Wilcoxon rank sum tests where used when data deviated from normality. Results are shown in corresponding boxplots (Figure 2 for cuff measurements and Figure 3 for PAD imaging). $\mathrm{P}$ values $<0.05$ were considered to be statistically significant. Reported results are represented as the mean value \pm one standard deviation.

\section{Results}

\section{Cuff-induced arterial occlusion measurements}

First, we scanned the calf muscle of four healthy volunteers during a 3-minute cuff-induced arterial occlusion, to investigate and demonstrate the capability of MSOT in monitoring skeletal muscle perfusion and oxygenation changes over time (Figure 2). Figure $2 A$ illustrates the temporal fluctuations of $\mathrm{Hb}, \mathrm{HbO}_{2}, \mathrm{THb}$ and $\mathrm{SO}_{2}$ for a 

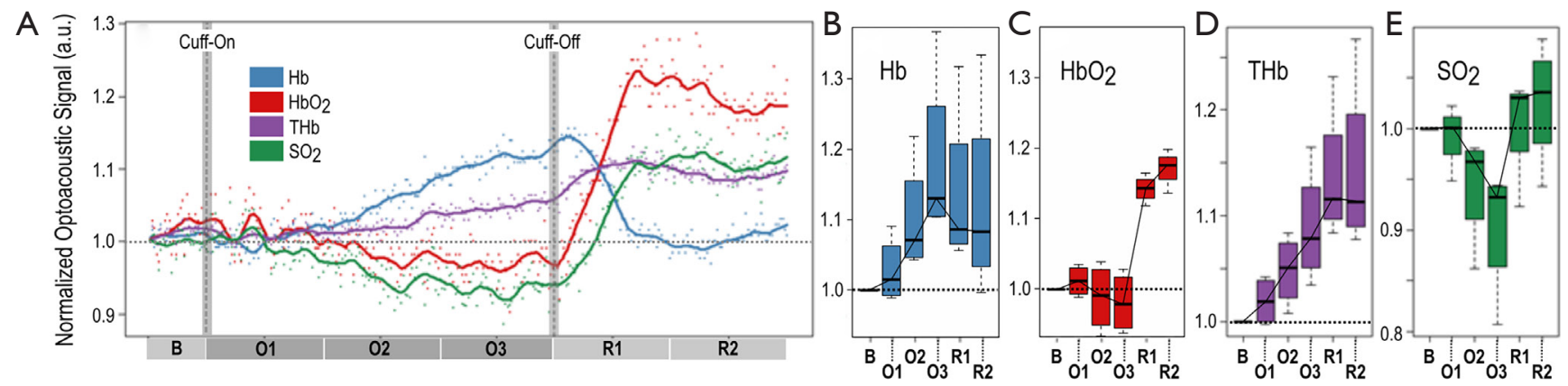

F
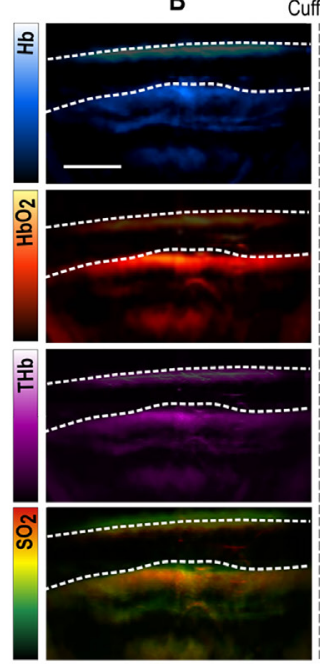

01
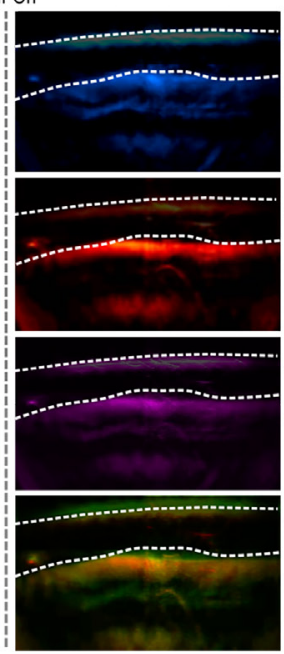

02
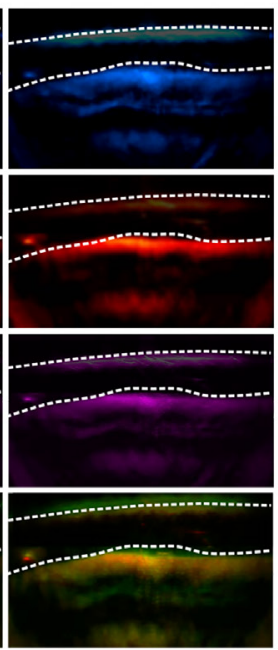

O3

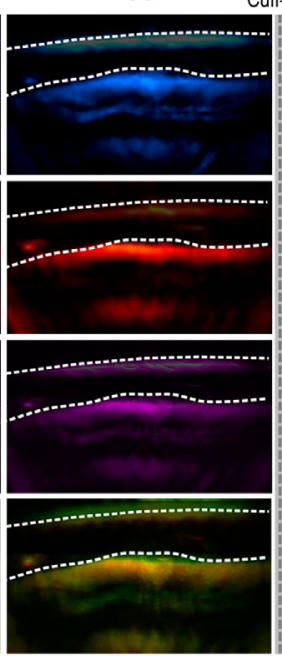

R1

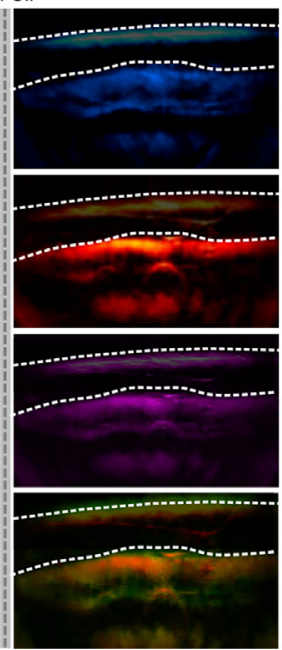

R2

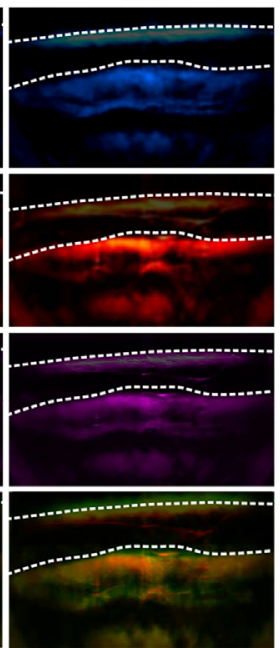

Figure 2 Cuff-induced arterial occlusion measurements. (A). Exemplary plots of $\mathrm{Hb}$ (deoxygenated hemoglobin), $\mathrm{HbO}_{2}$ (oxygenated hemoglobin), $\mathrm{THb}$ (total hemoglobin) and $\mathrm{SO}_{2}$ (oxygen saturation) within the calf skeletal muscle of a healthy volunteer. The blue line represents the $\mathrm{Hb}$ changes, the red line represents the $\mathrm{HbO}_{2}$ changes, the purple line the $\mathrm{THb}$ changes and the green line the $\mathrm{SO}_{2}$ changes over time. All three lines were normalized to their respective baseline values. B is the baseline period ( $30 \mathrm{~s}$ before the cuff inflation), O1 is the first $60 \mathrm{~s}$ of cuff occlusion, $\mathrm{O} 2$ is the next $60 \mathrm{~s}$ of cuff occlusion, $\mathrm{O} 3$ is the last $60 \mathrm{~s}$ of cuff occlusion, R1 is the first $60 \mathrm{~s}$ after cuff deflation and R2 is the next $60 \mathrm{~s}$ after the deflation of the cuff. Statistics for changes of (B) $\mathrm{Hb},(\mathrm{C}) \mathrm{HbO}_{2}$, (D) THb and (E) SO $\mathrm{T}_{2}$ within the calf muscles of all $\left(n_{1}=4\right)$ healthy volunteers for each time period described in (A). For the panels $(B, C, D, E)$, a subject is represented within the box of each time period by the average value of the plotted parameter during the specific time period. The images in (F) show the changes of intramuscular $\mathrm{Hb}\left(1^{\text {st }}\right.$ line $), \mathrm{HbO}_{2}\left(2^{\text {nd }}\right.$ line $), \mathrm{THb}\left(3^{\text {rd }}\right.$ line $)$ and $\mathrm{SO}_{2}$ ( $4^{\text {th }}$ line $)$ distribution for the same time periods and subject as in (A). Each image represents the last second of the corresponding time period. The color bars represent the range of the values for a specific parameter for the whole set of the depicted images (the maximum value is the maximum of the set of all image maxima, the minimum value is the minimum of the set of all image minima). Upper white dashed line: skin surface. Lower white dashed line: interface between subcutaneous fat and muscle or else muscle line. Muscle area: Image area below the muscle line. For all images, the intensity of the area above the muscle has been lightly suppressed for visualization purposes. The scale bar is $1 \mathrm{~cm}$.

healthy volunteer, as an example. Corresponding MSOT images of the same healthy volunteer as in Figure $2 \mathrm{~A}$, as well as the MSOT readouts across the entire cohort show a gradual increase of intramuscular $\mathrm{Hb}$ (Figure $2 B, F$ ) during the arterial occlusion period followed by a decrease after the release of the occlusion. Correspondingly, a gradual decrease in $\mathrm{HbO}_{2}$ (Figure 2C,F) is followed by a significant increase after the deflation of the cuff or else during the reactive hyperemia period. Furthermore, the THb (Figure $2 D, F)$ remains relatively stable with increasing trend over the occlusion period with an increase after flow release and $\mathrm{SO}_{2}$ (Figure $2 E, F$ ) follows the same fluctuation patters as that 

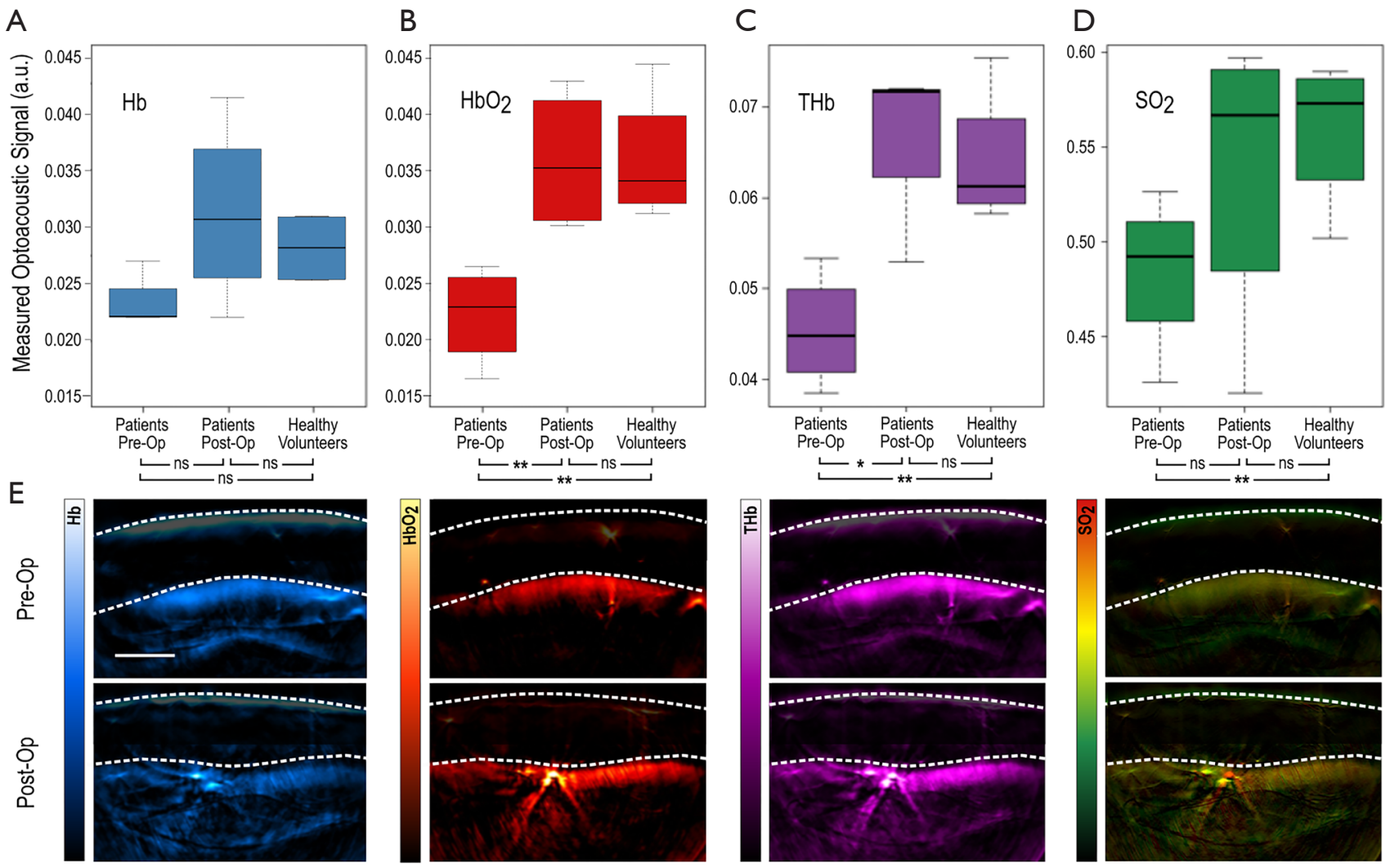

Figure 3 PAD measurements: comparison between patients and healthy volunteers as well as between patients before and patients after intervention. (A) Statistics for fluctuations in $\mathrm{Hb}$ (deoxygenated hemoglobin), (C) statistics for fluctuations in $\mathrm{HbO}_{2}$ (oxygenated hemoglobin), (D) statistics for fluctuations in THb (total hemoglobin) and (E) statistics for fluctuations $\mathrm{SO}_{2}$ (oxygen saturation) within the calf muscles of all $\left(n_{2}=4\right)$ patients with PAD before and after intervention, as well as, all $\left(n_{3}=4\right)$ healthy volunteers at rest. For the panels (A,B,C,D), each subject is represented by the average value of the plotted parameter within the calf muscle region as measured by means of MSOT. The exemplary images in (E) show the intramuscular $\mathrm{Hb}, \mathrm{HbO}_{2}, \mathrm{THb}$ and $\mathrm{SO}_{2}$ for a patient with PAD before ( $1^{\text {st }}$ line) and after $\left(2^{\text {nd }}\right.$ line) intervention. ns: statistically non-significant difference, *, $\mathrm{P}>0.05$ (marginally); ${ }^{* *}, \mathrm{P}<0.05$. The color bars represent the range of the values for a specific parameter for the both depicted images (the maximum value is the maximum of the both image maxima, the minimum value is the minimum of both image minima). The skeletal muscle region is the area below the white dashed line. For all images, the intensity of the area above the muscle has been lightly suppressed for visualization purposes. The scale bar is $1 \mathrm{~cm}$.

of $\mathrm{HbO}_{2}$ described above. Table 2 summarizes our findings regarding the cuff-induced arterial occlusion measurements.

\section{Measurements of patients with PAD}

Next, we used MSOT to examine the differences between healthy volunteers and patients with PAD, as well as the impact of the interventional revascularization therapy on muscle hemodynamics and oxygenation (Figure 3). We extracted and plotted the levels of $\mathrm{Hb}$ (Figure $3 A$ ), $\mathrm{HbO}_{2}$ (Figure $3 B$ ), $\mathrm{THb}$ (Figure $3 C$ ) and $\mathrm{SO}_{2}$ (Figure $3 D$ ) from the calf muscle ROIs of the MSOT images. Our results show statistically significant differences between healthy volunteers and $\mathrm{PAD}$ patients before intervention for $\mathrm{HbO}_{2}$ $(0.022 \pm 0.004$ for patients vs. $0.036 \pm 0.006$ for healthy, $\mathrm{P}=0.028), \mathrm{THb}(0.064 \pm 0.008$ vs. $0.045 \pm 0.006, \mathrm{P}=0.009)$ and $\mathrm{SO}_{2}(0.56 \pm 0.04$ vs. $0.484 \pm 0.042, \mathrm{P}=0.041)$. Furthermore, patients with PAD show statistically significant differences for $\mathrm{HbO}_{2}$ before and after treatment $(0.022 \pm 0.004$ before, $0.036 \pm 0.006$ after, $\mathrm{P}=0.029)$, while the change in $\mathrm{THb}$ before and after treatment is marginally significant or $(0.045 \pm 0.006$ before, $0.067 \pm 0.009$ after, $\mathrm{P}=0.057)$ and the 
Table 2 Percentage mean changes of $\mathrm{Hb}$ (deoxygenated hemoglobin), $\mathrm{HbO}_{2}$ (oxygenated hemoglobin), $\mathrm{THb}$ (total hemoglobin) and $\mathrm{SO}_{2}$ (oxygen saturation) over time with reference to the baseline during the cuff-induced arterial occlusion measurements for the entire group of volunteers $\left(\mathrm{n}_{1}=4\right)$

\begin{tabular}{|c|c|c|c|c|}
\hline Time & $\mathrm{Hb}$ & $\mathrm{HbO}_{2}$ & $\mathrm{THb}$ & $\mathrm{SO}_{2}$ \\
\hline $1^{\text {st }}$ minute of occlusion & $+2.7 \%$ & $+1.2 \%$ & $+1.9 \%$ & $-0.7 \%$ \\
\hline $2^{\text {nd }}$ minute of occlusion & $+9.9 \%$ & $-1.2 \%$ & $+4.8 \%$ & $-5.6 \%$ \\
\hline $3^{\text {rd }}$ minute of occlusion & $+18.1 \%$ & $-1.9 \%$ & $+8.9 \%$ & $-9.7 \%$ \\
\hline $1^{\text {st }}$ minute after cuff deflation & $+13.5 \%$ & $+14 \%$ & $+13.6 \%$ & $+0.5 \%$ \\
\hline
\end{tabular}

corresponding change in $\mathrm{SO}_{2}$ is non-significant $(\mathrm{P}=0.287)$. Finally, MSOT readouts show no significant difference for all measured parameters $\mathrm{HbO}_{2}, \mathrm{Hb}$, THb and $\mathrm{SO}_{2}$ between the groups of the healthy volunteers and the PAD patients after the application of the interventional treatment. Figure $3 E$ shows exemplary images of a patient with PAD preoperatively and postoperatively for all MSOT-extracted parameters.

\section{Discussion}

In this pilot study, we employed clinical MSOT hybridized with conventional ultrasound as a novel imaging technique for PAD assessment and therapy evaluation. PAD is a serious condition, that carries a substantial morbidity and is additionally considered to be an indicator for coronary artery disease and stroke $(24,25)$. PAD affects more than 200 million people worldwide (26), with significant restraints in life quality and huge healthcare costs. Currently established techniques for PAD imaging focus on the macrovascular pathology of atherosclerotic lesions, without providing any functional or metabolic assessment (e.g., perfusion or oxygenation) of the affected muscle. A timely assessment of 41,882 hospitalized PAD patients revealed that, especially in advanced stages of disease, a lack of appropriate diagnostics limits the use of revascularization procedure resulting in an unacceptable high rate of amputations (1). In detail, the study reports an amputation rate of $18.7 \%$ and an amputation risk of $59.6 \%$ within 1 year in patients with Rutherford stage 6 PAD.

Herein, we demonstrate that clinical hand-held MSOT can be used for extracting image-based biomarkers of muscle hemodynamics and oxygenation in patients with PAD. First, we show that MSOT is capable of detecting and quantifying the arterial occlusion-associated changes within muscle over time in real-time. Moreover, by sensing the reactive post-occlusive hyperemic response, MSOT may be developed as a novel tool for assessing microvascular endothelial dysfunction. MSOT can also produce high resolution maps of $\mathrm{Hb}$ and $\mathrm{HbO}_{2}$ deep in skeletal muscle over time, surpassing the information accessible using NIRS. Thus, MSOT could enable the definition of new image-based biomarkers as possible indicators of microvascular disease that appear before the manifestation of anatomical macrovascular changes in the affected limb, aiding the early identification of high-risk patients.

Second, we show that MSOT enables the differentiation between healthy volunteers and patients with PAD via direct and non-invasive muscle imaging without the need for exogenous contrast agents by extraction of perfusionand oxygenation-related functional tissue biomarkers. Thus, MSOT shows potential as a novel clinical tool for the detection of PAD and differentiation of other diseases causing similar clinical symptoms, such as spinal stenosis, peripheral neuropathy or deep vein thrombosis (27). This study focused on assessing muscle perfusion in healthy volunteers and PAD patients. However, skin perfusion might be also altered in PAD patients. Thus, further investigation of skin versus muscle perfusion in PAD might be promising in future studies. In this context, although MSOT/US has been used for skin and subcutaneous tissue assessment in diseases associated with microvascular dysfunction, dedicated optoacoustic techniques for skin imaging like raster-scan optoacoustic mesoscopy (RSOM) might be promising to assess skin perfusion in PAD patients.

Third, our results demonstrate that MSOT is able to visualize and quantify the effect of endovascular treatment on several functional parameters of muscle tissue perfusion. In our study patients with $\mathrm{PAD}$ showed, with an increase of about 1.6-fold, a significantly higher MSOT $\mathrm{HbO}_{2}$ value after than compared to before revascularization 
therapy. Although not fully comparable, the dimension of improvement is in line with other studies using for, e.g., thermal load tests with transcutaneous oxygen tension $\left(\mathrm{tc} \mathrm{PO}_{2}\right)$ measurement observing an improvement of 1.3fold to up to 11 -fold $(28,29)$ or of NIRS measurements showing an $\mathrm{HbO}_{2}$ improvement of about 1.8-fold (30). Thus, MSOT-extracted parameters may well be used for the objective quantification of different therapeutic effects.

Nevertheless, even if MSOT offers rich functional information via hemoglobin-based molecular contrast, this information should be evaluated with conventional imaging techniques. For example, our clinical hybrid MSOTUS scanner facilitates such a combination by overlaying functional MSOT data with anatomical information from US. Such system configurations open up excellent possibilities for revealing correlations between MSOT and other imaging modalities in order to promote its clinical translation as a hand-held molecular imaging technique.

MSOT technology does not come without limitations. Even with unprecedented penetration depths compared to purely optical methods, the depth achieved $(3-4 \mathrm{~cm})$ is still poor compared to traditional modalities used for PAD imaging in everyday clinical practice (US, MRA, CTA). The development of new illumination regimes or schemes for light attenuation with depth is expected to address these limitations in the future. Furthermore, the spectral unmixing step is conducted on a per-pixel basis and is thus vulnerable to motion artifacts, which may be further emphasized by noise due to vessel reactivity. For this reason, several motion correction algorithms have been recently introduced $(31,32)$. Further, a recent study showed that MSOT provided consistent and reproducible functional soft tissue characterization, independent on the investigating personnel (33). However, robustness and reproducibility of MSOT should be further investigated in future studies.

Notably, our pilot clinical study is limited by a low patient number. Future studies are expected to test the specificity, sensitivity and accuracy of MSOT technology for diagnosis or therapy monitoring in PAD. Nevertheless, current results demonstrate the capability of MSOT as a portable, non-invasive, label- and radiation-free molecular imaging modality for quantitative imaging of functional biomarkers in PAD with novel implications for both basic research on PAD pathophysiology and clinical molecular imaging.

\section{Conclusions}

The potential use of MSOT for PAD opens up new possibilities to investigate muscle oxygenation and metabolism in PAD, and to shift the paradigm from only imaging the cause of the disease (arterial stenosis), towards imaging the target organ (muscle) for more objective PAD diagnostics, treatment selection and therapy assessment.

\section{Acknowledgments}

We thank Dr. Jing Claussen (iThera Medical GmbH, Munich, Germany) and Dr. Niklas Markwardt (Institute for Biological and Medical Imaging, Helmholtz Center Munich, Neuherberg, Germany) for the invaluable technical support and fruitful discussions.

Funding: This project has received funding from the European Research Council (ERC) under the European Union's Horizon 2020 research and innovation programme under grant agreement No. 694968 (PREMSOT), by the Helmholtz Zentrum München through Physician Scientists for Groundbreaking Projects, in part by the Helmholtz Association of German Research Center, through the Initiative and Networking Fund, i3 (ExNet-0022-Phase2-3) and was supported by the DZHK (German Centre for Cardiovascular Research).

\section{Footnote}

Data Sharing Statement: Available at http://dx.doi. org/10.21037/atm-20-3321

Conflicts of Interest: All authors have completed the ICMJE uniform disclosure form (available at http://dx.doi. org/10.21037/atm-20-3321). Prof. VN reports to be a stock owner of iThera Medical GmbH, Munich, Germany. The other authors have no conflicts of interest to declare.

Ethical Statement: The authors are accountable for all aspects of the work in ensuring that questions related to the accuracy or integrity of any part of the work are appropriately investigated and resolved. The study was conducted in accordance with the Declaration of Helsinki (as revised in 2013). All participants gave written and informed consent prior to study enrolment. Healthy volunteers freely consented to participate in full accordance with the work safety regulations of the Helmholtz Center Munich (Neuherberg, Germany). The study was reviewed by the local ethics committee (Ethikkommission der Westfälischen WilhelmsUniversität Münster, Protocol No 2017-538-f-S). 
Open Access Statement: This is an Open Access article distributed in accordance with the Creative Commons Attribution-NonCommercial-NoDerivs 4.0 International License (CC BY-NC-ND 4.0), which permits the noncommercial replication and distribution of the article with the strict proviso that no changes or edits are made and the original work is properly cited (including links to both the formal publication through the relevant DOI and the license). See: https://creativecommons.org/licenses/by-nc-nd/4.0/.

\section{References}

1. Reinecke H, Unrath M, Freisinger E, et al. Peripheral arterial disease and critical limb ischaemia: still poor outcomes and lack of guideline adherence. Eur Heart J 2015;36:932-8.

2. Eberhardt RT, Coffman JD. Cardiovascular morbidity and mortality in peripheral arterial disease. Curr Drug Targets Cardiovasc Haematol Disord 2004;4:209-17.

3. Aday AW, Kinlay S, Gerhard-Herman MD. Comparison of different exercise ankle pressure indices in the diagnosis of peripheral artery disease. Vasc Med 2018;23:541-8.

4. Pollak AW, Norton PT, Kramer CM. Multimodality imaging of lower extremity peripheral arterial disease: current role and future directions. Circ Cardiovasc Imaging 2012;5:797-807.

5. Harvey C. Ultrasound with microbubbles. Cancer Imaging 2015;15:O19.

6. Suo S, Zhang L, Tang H, et al. Evaluation of skeletal muscle microvascular perfusion of lower extremities by cardiovascular magnetic resonance arterial spin labeling, blood oxygenation level-dependent, and intravoxel incoherent motion techniques. J Cardiovasc Magn Reson 2018;20:18.

7. Jones S, Chiesa ST, Chaturvedi N, et al. Recent developments in near-infrared spectroscopy (NIRS) for the assessment of local skeletal muscle microvascular function and capacity to utilise oxygen. Artery Res 2016;16:25-33.

8. Keller E, Nadler A, Alkadhi H, et al. Noninvasive measurement of regional cerebral blood flow and regional cerebral blood volume by near-infrared spectroscopy and indocyanine green dye dilution. Neuroimage 2003;20:828-39.

9. Khalil MA, Kim HK, Hoi JW, et al. Detection of Peripheral Arterial Disease Within the Foot Using Vascular Optical Tomographic Imaging: A Clinical Pilot Study. Eur J Vasc Endovasc Surg 2015;49:83-9.

10. Karlas A, Reber J, Diot G, et al. Flow-mediated dilatation test using optoacoustic imaging: a proof-of-concept. Biomedical Optics Express 2017;8:3395-403.

11. Yang H, Jüstel D, Prakash J, et al. Soft ultrasound priors in optoacoustic reconstruction: Improving clinical vascular imaging. Photoacoustics 2020;19:100172.

12. Karlas A, Fasoula NA, Paul-Yuan K, et al. Cardiovascular optoacoustics: From mice to men - A review. Photoacoustics 2019;14:19-30.

13. Karlas A, Kallmayer M, Fasoula NA, et al. Multispectral Optoacoustic Tomography of Muscle Perfusion and Oxygenation under Arterial and Venous Occlusion: A Human Pilot Study. J Biophotonics 2020;13:e201960169.

14. Taruttis A, Ntziachristos V. Advances in real-time multispectral optoacoustic imaging and its applications. Nat Photonics 2015;9:219-27.

15. Masthoff M, Helfen A, Claussen J, et al. Use of Multispectral Optoacoustic Tomography to Diagnose Vascular Malformations. JAMA Dermatol 2018;154:1457-62.

16. Masthoff M, Helfen A, Claussen J, et al. Multispectral optoacoustic tomography of systemic sclerosis. J Biophotonics 2018;11:e201800155.

17. Reber J, Willershäuser M, Karlas A, et al. Non-invasive Measurement of Brown Fat Metabolism Based on Optoacoustic Imaging of Hemoglobin Gradients. Cell Metabolism 2018;27:689-701.e4.

18. Roll W, Markwardt NA, Masthoff M, et al. Multispectral optoacoustic tomography of benign and malignant thyroid disorders: a pilot study. J Nucl Med 2019;60:1461-6.

19. Diot G, Metz S, Noske A, et al. Multispectral Optoacoustic Tomography (MSOT) of Human Breast Cancer. Clin Cancer Res 2017;23:6912-22.

20. Knieling F, Neufert C, Hartmann A, et al. Multispectral Optoacoustic Tomography for Assessment of Crohn's Disease Activity. N Engl J Med 2017;376:1292-4.

21. Becker A, Masthoff M, Claussen J, et al. Multispectral optoacoustic tomography of the human breast: characterisation of healthy tissue and malignant lesions using a hybrid ultrasound-optoacoustic approach. Eur Radiol 2018;28:602-9.

22. Ansi Z. American National Standard for Safe Use of Lasers. Laser Institute of America, Orlando; 2000.136.1.

23. Rosenthal A, Ntziachristos V, Razansky D. Model-based optoacoustic inversion with arbitrary-shape detectors. Med Phys 2011;38:4285-95.

24. Grenon SM, Vittinghoff E, Owens CD, et al. Peripheral artery disease and risk of cardiovascular events in patients with coronary artery disease: Insights from the Heart and 
Soul Study. Vasc Med 2013;18:176-84.

25. Kolls BJ, Sapp S, Rockhold FW, et al. Stroke in Patients With Peripheral Artery Disease. Stroke 2019;50:1356-63.

26. Shu J, Santulli G. Update on peripheral artery disease: Epidemiology and evidence-based facts. Atherosclerosis 2018;275:379-81.

27. Weinberg I, Jaff MR. Nonatherosclerotic arterial disorders of the lower extremities. Circulation 2012;126:213-22.

28. Katsui S, Inoue Y, Yamamoto Y, et al. In Patients with Severe Peripheral Arterial Disease, RevascularizationInduced Improvement in Lower Extremity Ischemia Can Be Detected by Laser Speckle Contrast Imaging of the Fluctuation in Blood Perfusion after Local Heating. Ann Vasc Surg 2018;48:67-74.

29. Yamamoto Y, Inoue Y, Igari K, et al. Assessment of the Severity of Ischaemia and the Outcomes of Revascularisation in Peripheral Arterial Disease Patients Based on the Skin Microcirculatory Response to a

Cite this article as: Karlas A, Masthoff M, Kallmayer M, Helfen A, Bariotakis M, Fasoula NA, Schäfers M, Seidensticker M, Eckstein HH, Ntziachristos V, Wildgruber M. Multispectral optoacoustic tomography of peripheral arterial disease based on muscle hemoglobin gradients-a pilot clinical study. Ann Transl Med 2021;9(1):36. doi: 10.21037/atm-203321
Thermal Load Test. EJVES Short Rep 2019;42:21-5.

30. Manfredini F, Lamberti N, Ficarra V, et al. Biomarkers of Muscle Metabolism in Peripheral Artery Disease: A Dynamic NIRS-Assisted Study to Detect Adaptations Following Revascularization and Exercise Training. Diagnostics (Basel) 2020;10:312.

31. Ulrich L, Held KG, Jaeger M, et al. Reliability assessment for blood oxygen saturation levels measured with optoacoustic imaging. J Biomed Opt 2020;25:1-15.

32. Jeng GS, Li ML, Kim M, et al. Real-time spectroscopic photoacoustic/ultrasound (PAUS) scanning with simultaneous fluence compensation and motion correction for quantitative molecular imaging. bioRxiv 2019. doi: 10.1101/2019.12.20.885251.

33. Helfen A, Masthoff M, Claussen J, et al. Multispectral Optoacoustic Tomography: Intra- and Interobserver Variability Using a Clinical Hybrid Approach. J Clin Med 2019;8:63. 